\title{
Response to "Anatomy of an internet consultation"
}

Dear Sir,

My heartfelt gratitude to the association and to you for keeping me on your mailing list. Though I retired 10 years ago, I try to keep up with the new innovations and ideas by reading journals. I specially enjoy the Indian 
Journal of Plastic Surgery because it keeps me updated about the current status of this blend of special art and science in India, the country where plastic surgery started centuries ago.

I enjoyed the note by Dr. A. Nirale: "Anatomy of an internet consultation" in the December issue. The ideas put up by the author are great. I would like to comment on the safety tips on page 197. The tip number 5 is a good tip but it should be expanded. Any comments if adverse and not complementing the "other" surgeon should be kept strictly to the second opinion surgeon. We often say things about other person's result which comes back to hound us. In this environment of malpractice cases filed for trivial causes, it is best not to comment about results as seen in photographs or in person.

I was impressed by other articles including the one about cleft surgery in India by Adenwalla et al. I was happy to see the name of Prof. Sam Bose in it. I first met him at the International Congress in Paris in summer of 1975. He introduced me to the Association of Plastic Surgeons of India and to ASI. I used to attend the meetings every December and gave several talks in the 70s and early 80 s. I went to Madurai to see this great man the day Tsunami hit India. He was bright and jovial as usual.

\section{N. K. Pandeya}

Clinical Prof. of Plastic Surgery (retired) at Des Moines University, College of Osteopathic Medicine, Des Moines, lowa and Kirksville College of Osteopathic Medicine, Kirksville, Missouri Brigadier General (retired), lowa Air National Guard

\section{The extended uses of Foley catheter in Plastic Surgery}

Dear Sir,

After modification of the urinary catheter by the addition of an inflatable distal retaining balloon by Dr. Frederick E. B. Foley in 1934, the simplicity of the resulting device has led to diverse applications in many fields of surgery. The Foley's catheter has been used by almost all surgeons at least one time in their practice. We present the extended uses of the Foley catheters in Plastic Surgery:

- Control of bleeding: Foley catheter has been used to provide balloon tamponade of major bleeding from the nasopharynx, maxillary, neck and supraclavicular fossae..$^{[1-4]}$

- Reduction of the facial fractures: Foley catheter has been used for orbital, unstable zygomatic arch and corpus fractures. ${ }^{[5,6]}$

- Reconstruction of the orbital floor: A Foley catheter was placed into the maxillary sinus to provide temporary support for the lyophilized tensor fascia lata graft..$^{[7]}$

- Eye socket reconstruction: Foley's catheter has been used for 2 purposes in this situation: to keep the skin bag bulging and to drain the exudates in the skin bag. ${ }^{[8]}$

- Scrotal reconstruction: Foley's catheters have been placed subcutaneously in the perineoscrotal region and skin has been slowly expanded for secondary scrotal reconstruction..$^{[9]}$

- Vaginal reconstruction: Foley's catheter has been used as a part of the soft vaginal mould for McIndoe's vaginoplasty. ${ }^{[10]}$

- Intraoperative tissue expansion: Tissue gain has been achieved by intraoperative tissue expansion using Foley's catheter. For this aim Foley's catheter has been used for cleft palate repair, eyelid reconstruction and some skin defects. ${ }^{[11-14]}$

- Experimental tissue expansion: A method of tissue expansion with a siliconized Foley's catheter has been described for experimental studies. ${ }^{[15]}$

- Alar stabilization: A technique of intraoperative nasal ala stabilization to aid excision of skin lesions on the nasal ala and the surrounding skin has been described. ${ }^{[16]}$

- Staged tendon reconstruction: Siliconized urinary catheter has been used experimentally as a silicone rod implant in staged tendon reconstruction. ${ }^{[17]}$

- Nostril retainer: A part of silicone urine catheter has been used as a nostril retainer. ${ }^{[18]}$

- Air drill irrigation: Foley's catheter has been used for intraoperative air drill burr irrigation. ${ }^{[19]}$

- Short-term drainage in frontal sinus surgery: Foley's catheter has been used purpose a short-term drainage and fronto-nasal duct kept patent for several weeks or months of frontal sinus disease. ${ }^{[20]}$

- In patients whom sustained full-thickness penis burns, foley catheter has been used scaffold for urethral sheet grafts and penile traction with a weighted catheter. ${ }^{[21]}$

In addition of these extended uses, we believe that there are many unpublished uses of the Foley's catheter in Plastic Surgery. 